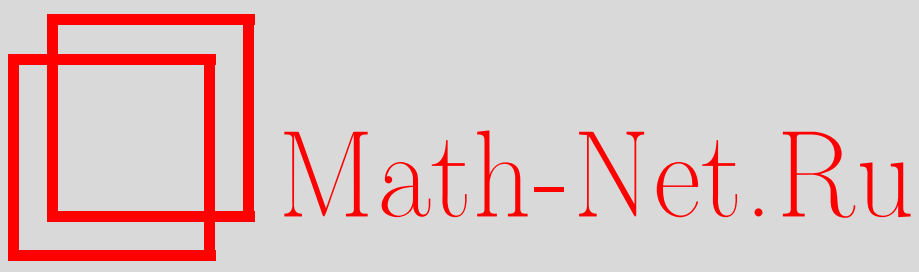

Г. Г. Лаптев, Отсутствие решений дифференциальных неравенств и систем гиперболического типа в конических областях, Изв. РАН. Сер. матем., 2002, том 66, выпуск 6, 65-90

DOI: https://doi.org/10.4213/im410

Использование Общероссийского математического портала Math-Net.Ru подразумевает, что вы прочитали и согласны с пользовательским соглашением

http://www.mathnet.ru/rus/agreement

Параметры загрузки:

IP : 44.207 .124 .84

26 апреля 2023 г., 18:33:03 
УДК 517.9

\author{
Г.Г. Лаптев
}

\title{
Отсутствие решений дифференциальных неравенств и систем гиперболического типа в конических областях
}

\footnotetext{
Установлены достаточные условия отсутствия глобальных решений полулинейных гиперболических неравенств и систем в конических областях евклидового пространства $\mathbb{R}^{N}$. В качестве модельной задачи в конусе $K$ рассматривается неравенство

$$
\frac{\partial^{2} u}{\partial t^{2}}-\Delta u \geqslant|u|^{q}, \quad(x, t) \in K \times(0, \infty),
$$

с неотрицательными начально-краевыми условиями.

Доказательство основано на методе пробных функций, разработанном Л. Вероном, Э. Митидиери, С.И. Похожаевым и А. Тесеем.

Библиография: 23 наименования.
}

\section{Введение}

Рассмотрим вопросы отсутствия глобальных решений полулинейных гиперболических неравенств и систем в случае, когда область (по пространственной переменной $x$ ) есть конус.

Проблема отсутствия глобальных решений эволюционных задач активно исследуется в настоящее время, в частности, благодаря книге [1]. Вопросам отсутствия решений для гиперболических неравенств посвящено большое количество литературы. (Хорошим введением в тему является книга [2], обзор литературы см. в [3], [4].)

В качестве модельной естественно рассматривать задачу

$$
\begin{aligned}
\frac{\partial^{2} u}{\partial t^{2}}-\Delta u & =|u|^{q} \quad \text { в } \quad \mathbb{R}^{N} \times(0, \infty), \\
u(x, 0) & =u_{0}(x), \\
\frac{\partial u}{\partial t}(x, 0) & =u_{1}(x) \geqslant 0 \quad \text { в } \quad \mathbb{R}^{N},
\end{aligned}
$$

где $\Delta$ - оператор Лапласа. В отличие от эллиптических и параболических задач (см., например, [5], [6]) соответствующие гиперболические уравнения изучены сушественно меньше, в частности из-за наличия двух постановок задачи, которые требуют разных подходов к исследованию.

Работа выполнена при финансовой поддержке INTAS (проект № 971-30551), программы "Ведущие научные школы" (проект № 00-15-96047) и Российского фонда фундаментальных исследований (грант № 01-01-00884). 
В первой постановке рассматриваются задачи с начальными данными, имеющими компактный носитель. Классическим здесь является результат Джона [7]: задача $(0.1)$ в пространстве $\mathbb{R}^{3}$ не имеет глобального решения, если $1<q<q_{c}=1+\sqrt{2}$, ни для каких начальных данных. В дальнейшем было показано [8], что решения нет и в критическом случае $q=q_{c}$. Если $q>q_{c}$, то для некоторого класса начальных данных требуемое глобальное решение сушествует (это оправдывает название "критический показатель"). Критический показатель для произвольной размерности $N$ является положительным корнем квадратного уравнения

$$
(N-1) q^{2}-(N+1) q-2=0
$$

и был найден в работах [9] и [10]. При внешней простоте идей этих работ техническая сложность их реализации привела к тому, что к настоящему времени изучены лишь достаточно простые системы полулинейных уравнений, причем доказательство отсутствия решения в критическом случае $q_{c}$ является предметом отдельного исследования (см. [11] для случая $N=3$, а для случая $N \geqslant 4$ этот вопрос открыт).

Вторая постановка предполагает, что начальные данные могут иметь некомпактный носитель. Разумеется, получаемые условия применимы и к предыдущей задаче, но в силу большей общности критический показатель меньше соответствующего показателя Джона. Первые результаты для второй постановки были получены Като [12]: при $1<q \leqslant \frac{N+1}{N-1}$ задача (0.1) не имеет глобального решения. Точнее, в указанной работе начальные данные имеют компактный носитель, поскольку только в этом случае можно использовать предложенньй метод сведения к обыкновенному дифференциальному уравнению, однако полученный критический показатель является неулучшаемым именно в классе задач с некомпактными начальными данными [13].

В силу простоты метода Като доказаны аналогичные результаты для полулинейных систем с достаточно общими операторами в главной части (см., в частности, [10]) в предположении компактности начальных данных и конечной скорости распространения возмушений.

Следуюшим важным шагом стало применение к исследованию метода пробных функций [14]-[17], которому мы следуем и в настоящей работе. Этот метод является более общим, чем способ сведения к обыкновенному дифференциальному уравнению, и позволяет рассматривать задачи с некомпактными начальными данными [13]. Очень важным здесь является тот факт, что в действительности доказательство отсутствия решения основано на размерностных соображениях и не использует тип оператора, поэтому термин "гиперболические" применяется условно и в методе не используются какие бы то ни были сведения из линейной теории соответствующих гиперболических задач.

Метод пробных функций позволяет достаточно легко получать зависящие от параметра априорные оценки решений. Из стремления оценки к нулю следует отсутствие нетривиального решения без всяких предположений о поведении его на бесконечности. В то же время, если имеются некоторые ограничения на поведение 
решения или начальных данных (в частности, оценки снизу), метод также позволяет получать различные типы теорем отсутствия, которые в определенном смысле являются неулучшаемыми.

В настоящей статье мы рассматриваем дифференциальные неравенства, однако результаты об отсутствии решений справедливы, очевидно, и для соответствующих уравнений.

Пусть $K_{\omega}$ - область на единичной сфере $S^{N-1} \subset \mathbb{R}^{N}, N \geqslant 3$, с кусочно гладкой границей $\partial K_{\omega}$. Конусом $K$ называется множество, которое в сферической системе координат $(r, \omega), 0 \leqslant r<\infty, \omega \in S^{N-1}$, имеет вид

$$
K=\left\{x=(r, \omega): 0<r<+\infty, \omega \in K_{\omega}\right\} .
$$

Боковую поверхность конуса обозначим $\partial K$. Под конической областью $K_{R}$ понимается область $\{x \in K:|x|>R\}$ с полной границей $\partial K_{R}$. Заметим, что проводимые далее построения и используемые характеристики тесно связаны с исследованием линейных задач в окрестности конической точки границы [18], [19].

Через $\Delta$ обозначим оператор Лапласа по пространственным переменным $\left(x_{1}, \ldots, x_{N}\right)$, вектор из частных производных обозначим $D u=\left(\frac{\partial u}{\partial x_{i}}\right)$. Для двух дифференцируемых функций $u(x)$ и $\varphi(x)$ положим

$$
D u D \varphi=\sum_{i=1}^{N} \frac{\partial u}{\partial x_{i}} \frac{\partial \varphi}{\partial x_{i}} .
$$

Выражение $\frac{\partial u}{\partial n}$ обозначает производную функции $u$ по направлению внешней нормали $n$ к границе конуса $\partial K$. Через $c$ и $C$ с индексами будем обозначать постоянные.

Напомним, что оператор Лапласа $\Delta$ в сферических координатах $(r, \omega)$ имеет вид

$$
\Delta=\frac{1}{r^{N-1}} \frac{\partial}{\partial r}\left(r^{N-1} \frac{\partial}{\partial r}\right)+\frac{1}{r^{2}} \Delta_{\omega}=\frac{\partial^{2}}{\partial r^{2}}+\frac{N-1}{r} \frac{\partial}{\partial r}+\frac{1}{r^{2}} \Delta_{\omega}
$$

где $\Delta_{\omega}$ - оператор Бельтрами-Лапласа на единичной сфере $S^{N-1} \subset \mathbb{R}^{N}$.

В дальнейшем постоянно используются наименьшее (первое) собственное значение $\lambda_{\omega} \equiv \lambda_{1}\left(K_{\omega}\right)>0$ и соответствующая собственная функция $\Phi(\omega)$ оператора $\Delta_{\omega}$, являюшиеся решением задачи

$$
\begin{gathered}
\Delta_{\omega} \Phi+\lambda \Phi=0 \quad \text { в } K_{\omega}, \\
\left.\Phi\right|_{\partial K_{\omega}}=0 .
\end{gathered}
$$

Считаем $\Phi(\omega)>0$ для $\omega \in K_{\omega}$ и $\Phi(\omega) \leqslant 1$.

Пусть $\Omega$ - неограниченная область пространства $\mathbb{R}^{N+1}$ с кусочно гладкой границей. Далее используются пространства С. Л. Соболева $W_{q}^{2}(\Omega)$, а также локальное пространство $L_{q, \text { lос }}(\Omega)$, элементы которого принадлежат $L_{q}\left(\Omega^{\prime}\right)$ для любого компактного подмножества $\Omega^{\prime}: \bar{\Omega}^{\prime} \subset \Omega$. Через $C(\bar{\Omega})$ обозначается пространство непрерывных функций, через $C^{m}(\bar{\Omega})$ - пространство гладких функций на замкнутой области $\bar{\Omega}$. 


\section{§1. Вспомогательные построения}

Проводимые ниже построения имеют целью получение “срезки” функции, на которой рассматриваемый оператор неотрищателен, таким образом, чтобы она стала финитной по переменным $|x|=r$ и $t$. В случае всего пространства $\mathbb{R}^{N}$ удобной функцией является тождественная единица, однако для конуса необходимо использовать решения типа фундаментальных.

Будем искать решение уравнения Лапласа $\Delta v=0$ в конусе $K$ в виде $r^{s} \Phi(\omega)$. Вычисляя $\Delta\left(r^{s} \Phi(\omega)\right)$, с учетом равенства $\Delta_{\omega} \Phi=-\lambda_{\omega} \Phi$ приходим к выражению

$$
\Delta\left(r^{s} \Phi(\omega)\right)=r^{s-2} \Phi(\omega)\left\{s(s-1)+s(N-1)-\lambda_{\omega}\right\}
$$

Введем параметры

$$
s^{*}=\frac{N-2}{2}+\sqrt{\left(\frac{N-2}{2}\right)^{2}+\lambda_{\omega}}, \quad s_{*}=-\frac{N-2}{2}+\sqrt{\left(\frac{N-2}{2}\right)^{2}+\lambda_{\omega}} .
$$

Числа $s_{*}$ и $-s^{*}$ являются корнями многочлена, стояшего в фигурных скобках в предыдущем выражении для $\Delta\left(r^{s} \Phi(\omega)\right)$. Заметим, что $s^{*}-s_{*}=N-2$. Введем функцию на области $K_{R}$ :

$$
\xi(x) \equiv \xi(r, \omega)=\left(r^{s_{*}}-R^{s_{*}}\right) \Phi(\omega)
$$

Отметим ее свойства в $K_{R}$. Прежде всего $\Delta \xi \geqslant 0$, так как

$$
\begin{aligned}
\Delta \xi & =\Delta\left(\left(r^{s_{*}}-R^{s_{*}}\right) \Phi(\omega)\right) \\
& =r^{s_{*}-2} \Phi(\omega)\left\{s_{*}\left(s_{*}-1\right)+s_{*}(N-1)-\lambda_{\omega}\right\}-\frac{R^{s_{*}}}{r^{2}} \Delta_{\omega} \Phi \equiv \lambda_{\omega} \frac{R^{s_{*}}}{r^{2}} \Phi(\omega) \geqslant 0 .
\end{aligned}
$$

Далее, $\left.\xi\right|_{\partial K_{R}}=0,\left.\frac{\partial \xi}{\partial n}\right|_{\partial K_{R}} \leqslant 0$ (здесь $\frac{\partial \xi}{\partial n}-$ производная по внешней нормали $n$ к полной гранище конической области $\left.\partial K_{R}\right)$. Проверим последнее свойство. На боковой поверхности конуса

$$
\frac{\partial \xi}{\partial n}=\left(r^{s_{*}}-R^{s_{*}}\right) \frac{\partial \Phi(\omega)}{\partial n_{\omega}} \leqslant 0,
$$

где $n_{\omega}-$ внешняя нормаль к границе области $K_{\omega}$ (неположительность производной $\partial \Phi(\omega) / \partial n_{\omega}$ следует из леммы Хопфа). На участке $r=R$ получаем

$$
\left.\frac{\partial \xi}{\partial n}\right|_{r=R}=-\left.\frac{\partial \xi}{\partial r}\right|_{r=R}=-s_{*} R^{s_{*}-1} \Phi(\omega) \leqslant 0
$$

Основой последуюших построений является "стандартная срезающая функция" (см. [20]) $\zeta(y) \in C^{\infty}\left(\mathbb{R}_{+}\right)$со следующими свойствами:

$$
0 \leqslant \zeta(y) \leqslant 1, \quad \zeta(y)=\left\{\begin{array}{l}
1, \text { если } 0 \leqslant y \leqslant 1 \\
0, \text { если } y \geqslant 2
\end{array}\right.
$$


Введем также функцию $\eta(y)=(\zeta(y))^{2 p_{0}}$ с некоторым положительным параметром $p_{0}$. Тогда несложно проверить выполнение оценок (для $1<p \leqslant p_{0}$ )

$$
\begin{aligned}
\left|\eta^{\prime}(y)\right|^{p} & =\left(2 p_{0}\right)^{p} \zeta^{2 p_{0}(p-1)} \zeta^{2 p_{0}-p}\left|\zeta^{\prime}\right|^{p} \leqslant c_{\eta} \eta^{p-1}(y), \\
\left|\eta^{\prime \prime}(y)\right|^{p} & \leqslant\left(2 p_{0}\right)^{p} \zeta^{2 p_{0}(p-1)} \zeta^{2 p_{0}-2 p}\left(\left(2 p_{0}-1\right)\left|\zeta^{\prime}\right|^{2}+\zeta\left|\zeta^{\prime \prime}\right|\right)^{p} \leqslant c_{\eta} \eta^{p-1}(y)
\end{aligned}
$$

с некоторой постоянной $c_{\eta}$.

Вводя замену переменной $y=t / \rho^{\theta}$, где $\theta>0, \rho>2 R, t$-новая переменная, для функции $\eta\left(t / \rho^{\theta}\right)$, у которой

$$
\operatorname{supp}\left|\eta\left(\frac{t}{\rho^{\theta}}\right)\right|=\left\{t<2 \rho^{\theta}\right\}, \quad \operatorname{supp}\left|\frac{d^{2} \eta\left(t / \rho^{\theta}\right)}{d t^{2}}\right|=\left\{\rho^{\theta}<t<2 \rho^{\theta}\right\},
$$

легко установить неравенство

$$
\int_{\operatorname{supp}}\left|\frac{d^{2} \eta\left(t / \rho^{\theta}\right)}{d t^{2}}\right| \frac{\left|\frac{d^{2} \eta\left(t / \rho^{\theta}\right)}{d t^{2}}\right|^{p}}{\eta^{p-1}\left(t / \rho^{\theta}\right)} d t \leqslant c_{\eta} \rho^{-\theta(2 p-1)} .
$$

Параметр $\theta$ в дальнейшем будет подбираться в зависимости от оператора.

Соответственно, для пространственной переменной $x,|x|=r$, вводим функцию $\eta(r / \rho)$ и произведение

$$
\left(r^{s}-R^{s}\right) \eta\left(\frac{r}{\rho}\right)
$$

где в дальнейшем положим $s=s_{*}$. Получим оценки для первой и второй производных функции (1.4):

$$
\begin{aligned}
\left|\frac{\partial\left(\left(r^{s}-R^{s}\right) \eta(r / \rho)\right)}{\partial r}\right|^{p} & \leqslant\left|s r^{s-1} \eta\left(\frac{r}{\rho}\right)+\left(r^{s}-R^{s}\right) \eta^{\prime}\left(\frac{r}{\rho}\right) \frac{1}{\rho}\right|^{p} \\
& \leqslant c \eta^{p-1}\left(\frac{r}{\rho}\right) r^{p(s-1)}\left(1+\frac{r^{p}}{\rho^{p}}\right), \\
\left|\frac{\partial^{2}\left(\left(r^{s}-R^{s}\right) \eta(r / \rho)\right)}{\partial r^{2}}\right|^{p} & \leqslant\left|r^{s-2}\left(s(s-1) \eta\left(\frac{r}{\rho}\right)+2 s \eta^{\prime}\left(\frac{r}{\rho}\right) \frac{r}{\rho}+\eta^{\prime \prime}\left(\frac{r}{\rho}\right) \frac{r^{s}-R^{s}}{r^{s-2} \rho^{2}}\right)\right|^{p} \\
& \leqslant c \eta^{p-1}\left(\frac{r}{\rho}\right) r^{p(s-2)}\left(1+\frac{r^{p}}{\rho^{p}}+\frac{r^{2 p}}{\rho^{2 p}}\right),
\end{aligned}
$$

где константа $c$ не зависит от $r$ и $\rho$.

Теперь установим аналогичную (1.3) оценку для "срезанной" функции

$$
\psi_{\rho}(x)=\xi(x) \eta\left(\frac{|x|}{\rho}\right)=\left(r^{s_{*}}-R^{s_{*}}\right) \eta\left(\frac{r}{\rho}\right) \Phi(\omega) .
$$

С учетом выражений в сферических координатах

$$
\Delta \psi_{\rho}=\frac{\partial^{2} \psi_{\rho}}{\partial r^{2}}+\frac{N-1}{r} \frac{\partial \psi_{\rho}}{\partial r}+\frac{1}{r^{2}} \Delta_{\omega} \psi_{\rho}, \quad \Delta_{\omega} \psi_{\rho}=-\lambda_{\omega} \psi_{\rho}
$$


и установленных вьше оценок для производных от произведения $\left(r^{s}-R^{s}\right) \eta(r / \rho)$, которые используем для $s=s_{*}$, получим

$$
\begin{aligned}
\left|\Delta \psi_{\rho}(x)\right|^{p} & =\left|\left(\frac{\partial^{2}}{\partial r^{2}}+\frac{N-1}{r} \frac{\partial}{\partial r}-\frac{\lambda_{\omega}}{r^{2}}\right)\left(\left(r^{s_{*}}-R^{s_{*}}\right) \eta\left(\frac{r}{\rho}\right)\right)\right|^{p} \Phi^{p}(\omega) \\
& \leqslant c \eta^{p-1}\left(\frac{r}{\rho}\right) \Phi^{p}(\omega) \frac{r^{(p-1) s_{*}}}{r^{2 p-s_{*}}}\left(1+\frac{r^{p}}{\rho^{p}}+\frac{r^{2 p}}{\rho^{2 p}}\right) \\
& \leqslant c \psi_{\rho}^{p-1}(x) \frac{1}{r^{2 p-s_{*}}}\left(\frac{r^{s_{*}}}{r^{s_{*}}-R^{s_{*}}}\right)^{p-1}\left(1+\frac{r^{p}}{\rho^{p}}+\frac{r^{2 p}}{\rho^{2 p}}\right) .
\end{aligned}
$$

Поскольку $\eta(|x| / \rho) \equiv 1$ для $|x| \leqslant \rho$, то $\psi_{\rho}(x)=\xi(x)$ и $\Delta \psi_{\rho}=\Delta \xi \geqslant 0$, так что множество $K_{R}^{-} \equiv\left\{x: \Delta \psi_{\rho}<0\right\}$ содержится во множестве $\left\{K_{R} \cap\{\rho<|x|<2 \rho\}\right\}$, и на нем выполнено неравенство

$$
1+\frac{r^{p}}{\rho^{p}}+\frac{r^{2 p}}{\rho^{2 p}} \leqslant c
$$

где постоянная $c$ не зависит от $r$ и $\rho$. Кроме того, предположение $\rho \geqslant 2 R$ означает

$$
\frac{r^{s_{*}}}{r^{s_{*}}-R^{s_{*}}} \leqslant \frac{1}{1-(R / r)^{s_{*}}} \leqslant c
$$

следовательно,

$$
\left|\Delta \psi_{\rho}(x)\right|^{p} \leqslant c \psi_{\rho}^{p-1}(x) \rho^{s_{*}-2 p}
$$

Отсюда для любого параметра $\sigma \in \mathbb{R}$ вытекает оценка

$$
\begin{aligned}
\int_{\left\{x: \Delta \psi_{\rho}<0\right\}} \frac{\left|\Delta \psi_{\rho}(x)\right|^{p}}{\psi_{\rho}^{p-1}(x)|x|^{\sigma(p-1)}} d x & \leqslant c_{\sigma} \int_{\rho}^{2 \rho} \int_{K_{\omega}} \frac{\psi_{\rho}^{p-1}(x)}{\psi_{\rho}^{p-1}(x)} \frac{r^{N-1}}{\rho^{2 p-s_{*}+\sigma(p-1)}} d r d \omega \\
& \leqslant c_{\psi, \sigma} \rho^{-p(\sigma+2)+s_{*}+N+\sigma}
\end{aligned}
$$

Наконец, общую пробную функцию будем рассматривать в виде

$$
\varphi_{\rho}(x, t)=\eta\left(\frac{t}{\rho^{\theta}}\right) \psi_{\rho}(x)
$$

что с учетом полученных ранее оценок приводит к неравенству

$$
\begin{aligned}
& \iint_{\left\{x, t: \Delta \varphi_{\rho}<0\right\}} \frac{\left|\Delta \varphi_{\rho}(x, t)\right|^{p}}{\varphi_{\rho}^{p-1}(x, t)|x|^{\sigma(p-1)}} d x d t \\
& \quad \leqslant \int_{0}^{2 \rho^{\theta}} \eta\left(\frac{t}{\rho^{\theta}}\right) d t \int_{\left\{x: \Delta \psi_{\rho}<0\right\}} \frac{\left|\Delta \psi_{\rho}\right|^{p}}{\psi_{\rho}^{p-1}|x|^{\sigma(p-1)}} d x \\
& \quad \leqslant c_{\varphi} \rho^{\theta-p(\sigma+2)+s_{*}+N+\sigma} .
\end{aligned}
$$


Далее, используя неравенство (1.3), убедимся в том, что

$$
\begin{aligned}
& \iint_{\operatorname{supp} \mid}\left|\frac{\partial^{2} \varphi_{\rho}}{\partial t^{2}}\right| \frac{\left|\frac{\partial^{2} \varphi_{\rho}(x, t)}{\partial t^{2}}\right|^{p}}{\varphi_{\rho}^{p-1}(x, t)|x|^{\sigma(p-1)}} d x d t \\
& \quad \leqslant \int_{\operatorname{supp}\left|\frac{d^{2} \eta\left(t / \rho^{\theta}\right)}{d t^{2}}\right| \frac{\left|\frac{d^{2} \eta\left(t / \rho^{\theta}\right)}{d t^{2}}\right|^{p}}{\eta^{p-1}\left(t / \rho^{\theta}\right)} d t \int_{K_{R} \cap\{R<r<2 \rho\}} \frac{\psi_{\rho}(x)}{|x|^{\sigma(p-1)}} d x} \int_{K_{\omega}} \Phi(\omega) d \omega \int_{R}^{2 \rho}\left(r^{s_{*}}-R^{s_{*}}\right) \eta\left(\frac{r}{\rho}\right) \frac{r^{N-1}}{r^{\sigma(p-1)}} d r \\
& \leqslant c_{\eta} \rho^{-\theta(2 p-1)} \int_{\eta} \rho^{-\theta(2 p-1)}\left|K_{\omega}\right| \int_{R}^{2 \rho} r^{s_{*}} \frac{r^{N-1}}{r^{\sigma(p-1)}} d r \\
& \leqslant c_{\varphi} \cdot \begin{cases}\rho^{s_{*}+N-\sigma(p-1)-\theta(2 p-1)}, & \text { если } s_{*}+N-\sigma(p-1)>0, \\
\rho^{-\theta(2 p-1)} \ln \rho, & \text { если } s_{*}+N-\sigma(p-1)=0, \\
\rho^{-\theta(2 p-1)} R^{s_{*}+N-\sigma(p-1)}, & \text { если } s_{*}+N-\sigma(p-1)<0 .\end{cases}
\end{aligned}
$$

Из этой оценки для нас важным является первый показатель степени $\rho$. При $\theta=1$ этот показатель совпадает с показателем из (1.7), так как

$s_{*}+N-\sigma(p-1)-\left.\theta(2 p-1)\right|_{\theta=1}=\theta-p(\sigma+2)+s_{*}+N+\left.\sigma\right|_{\theta=1}=1-p(\sigma+2)+s_{*}+N+\sigma$.

Отметим важный для модельной задачи во всем конусе $K$ (когда $R=0$ ) частный случай $\sigma=0$. Тогда $\Delta \xi=0$ в $K$ и вместо множества $\left\{x: \Delta \psi_{\rho}<0\right\}$ можно рассматривать множество $\Delta \psi_{\rho} \neq 0$, т. е. $\operatorname{supp}\left|\Delta \psi_{\rho}\right|$. Далее, поскольку $\sigma=0$, то $s_{*}+N-\sigma(p-1) \equiv s_{*}+N>0$ и из неравенств (1.7) и (1.8) при $\theta=1$ получаем оценку

$$
\iint_{\operatorname{supp}\left|\frac{\partial^{2} \varphi_{\rho}}{\partial t^{2}}-\Delta \varphi_{\rho}\right|} \frac{\left|\frac{\partial^{2} \varphi_{\rho}}{\partial t^{2}}-\Delta \varphi_{\rho}\right|^{p}}{\varphi_{\rho}^{p-1}} d x d t \leqslant c_{0} \rho^{-2 p+s_{*}+N+1} .
$$

Пусть теперь $T>0$ фиксировано. Нам понадобятся аналогичные неравенствам (1.3), (1.7) и (1.8) оценки в области $t>T$ :

$$
\begin{aligned}
& \int_{\operatorname{supp}\left|\frac{d^{2} \eta\left(t / \rho^{\theta}\right)}{d t^{2}}\right|} \frac{\left|\frac{d^{2} \eta\left(t / \rho^{\theta}\right)}{d t^{2}}\right|^{p}}{\eta^{p-1}\left(t / \rho^{\theta}\right) t^{\gamma(p-1)}} d t \leqslant c_{\eta} \rho^{-\theta(p(2+\gamma)-1-\gamma)} ; \\
& \iint_{\left\{x, t: \Delta \varphi_{\rho}<0\right\}} \frac{\left|\Delta \varphi_{\rho}(x, t)\right|^{p}}{\varphi_{\rho}^{p-1}(x, t) t^{\gamma(p-1)}} d x d t \\
& \quad \leqslant \int_{T}^{2 \rho^{\theta}} \frac{\eta\left(t / \rho^{\theta}\right)}{t^{\gamma(p-1)} d t \int_{\left\{x: \Delta \psi_{\rho}<0\right\}} \frac{\left|\Delta \psi_{\rho}\right|^{p}}{\psi_{\rho}^{p-1}} d x} \\
& \leqslant c_{\varphi} \cdot \begin{cases}\rho^{-\gamma \theta(p-1)+\theta-2 p+s_{*}+N}, & \text { если } 1-\gamma(p-1)>0, \\
\rho^{-2 p+s_{*}+N} \ln \rho, & \text { если } 1-\gamma(p-1)=0, \\
\rho^{-2 p+s_{*}+N} T^{1-\gamma(p-1)}, & \text { если } 1-\gamma(p-1)<0 ;\end{cases}
\end{aligned}
$$




$$
\begin{aligned}
& \iint_{\operatorname{supp} \mid}\left|\frac{\partial^{2} \varphi_{\rho}}{\partial t^{2}}\right| \frac{\left|\frac{\partial^{2} \varphi_{\rho}(x, t)}{\partial t^{2}}\right|^{p}}{\varphi_{\rho}^{p-1}(x, t) t^{\gamma(p-1)}} d x d t \\
& \quad \leqslant \int_{\operatorname{supp}\left|\frac{d^{2} \eta\left(t / \theta^{\theta}\right)}{d t^{2}}\right|} \frac{\left|\frac{d^{2} \eta\left(t / \rho^{\theta}\right)}{d t^{2}}\right|^{p}}{\eta^{p-1}\left(t / \rho^{\theta}\right) t^{\gamma(p-1)}} d t \int_{K_{R} \cap\{R<r<2 \rho\}} \psi_{\rho}(x) d x \\
& \quad \leqslant c_{\varphi} \rho^{-\theta(p(2+\gamma)-1-\gamma)+s_{*}+N} .
\end{aligned}
$$

При $\theta=1$ выполнено

$-\gamma \theta(p-1)+\theta-2 p+s_{*}+N=-\theta(p(2+\gamma)-1-\gamma)+s_{*}+N=-p(2+\gamma)+\gamma+s_{*}+N+1$.

\section{§2. Модельная задача в конусе}

Рассмотрим проблему отсутствия слабых решений задачи

$$
\begin{gathered}
\frac{\partial^{2} u}{\partial t^{2}}-\Delta u \geqslant|u|^{q} \quad \text { в } K \times(0, \infty), \\
\left.u\right|_{\partial K \times[0, \infty)} \geqslant 0, \\
\left.\frac{\partial u}{\partial t}\right|_{t=0} \geqslant 0, \quad u \neq 0,
\end{gathered}
$$

которая используется как модельная задача в большинстве работ. Насколько известно автору, в конусе задача не рассматривалась и приводимый ниже результат является новым.

Далее слабое решение будет пониматься в следуюшем смысле.

ОПРЕДЕЛЕНИЕ 2.1. Пусть $u(x, t) \in C(\bar{K} \times[0, \infty))$ и определен локально суммируемый след $\frac{\partial u}{\partial t}$ при $t=0$. Функция $u(x, t)$ называется слабым решением за$\partial а ч и(2.1)$, если для любой неотрищательной пробной функции $\varphi(x, t) \in W_{\infty}^{2}(K \times$ $(0, \infty))$ такой, что $\left.\varphi\right|_{\partial K \times(0, \infty)}=0$, и финитной по переменным $r=|x|_{\text {и }} t$, выполнено интегральное неравенство

$$
\begin{aligned}
& \int_{0}^{\infty} \int_{\partial K} u \frac{\partial \varphi}{\partial n} d x d t+\int_{0}^{\infty} \int_{K} u\left(\frac{\partial^{2} \varphi}{\partial t^{2}}-\Delta \varphi\right) d x d t \\
& \quad \geqslant \int_{0}^{\infty} \int_{K}|u|^{q} \varphi d x d t-\int_{K} u(x, 0) \frac{\partial \varphi}{\partial t}(x, 0) d x+\int_{K} \frac{\partial u}{\partial t}(x, 0) \varphi(x, 0) d x
\end{aligned}
$$

TeOpema 2.1. $\Pi p u$

$$
1<q \leqslant q^{*}=1+\frac{2}{s^{*}+1}
$$

задача (2.1) не имеет нетривиального глобального решения. 
ЗАмечАниЕ 2.1. Для рассмотрения задачи (2.1) в пространстве $\mathbb{R}^{N}$ нужно взять $s^{*}=N-2$. Тогда приходим к классическому показателю Като:

$$
q^{*}=\frac{N+1}{N-1}
$$

ДокАЗАТЕльСтво проведем от противного. Пусть $u(x, t)$ - нетривиальное решение задачи (2.1) с $1<q \leqslant q^{*}$. Согласно определению 2.1 с пробной функцией $\varphi(x, t)=\varphi_{\rho}(x, t)$, введенной по формуле (1.6) при $p=q^{\prime}>1$ и $\theta=1$, имеем

$$
\begin{aligned}
\int_{K} \frac{\partial u}{\partial t}(x, 0) \varphi_{\rho}(x, 0) d x-\int_{K} u(x, 0) \frac{\partial \varphi_{\rho}}{\partial t}(x, 0) d x+\int_{0}^{\infty} \int_{K}|u|^{q} \varphi_{\rho} d x d t \\
\leqslant \int_{0}^{\infty} \int_{\partial K} u \frac{\partial \varphi_{\rho}}{\partial n} d x d t+\iint_{\operatorname{supp}\left|\frac{\partial^{2} \varphi_{\rho}}{\partial t^{2}}-\Delta \varphi_{\rho}\right|} u\left(\frac{\partial^{2} \varphi_{\rho}}{\partial t^{2}}-\Delta \varphi_{\rho}\right) d x d t
\end{aligned}
$$

Второй интеграл в левой части равен нулю. Производная функции $\varphi_{\rho}$ по нормали к границе конуса неположительна, поэтому первый интеграл в правой части будет неположительным благодаря условию $\left.u\right|_{\partial K \times[0, \infty)} \geqslant 0$.

Для оценки последнего интеграла в (2.2) применим неравенство Гёльдера. Получим

$$
\begin{aligned}
& \int_{K} \frac{\partial u}{\partial t}(x, 0) \varphi_{\rho}(x, 0) d x+\int_{0}^{\infty} \int_{K}|u|^{q} \varphi_{\rho} d x d t=\int_{K} \frac{\partial u}{\partial t}(x, 0) \varphi_{\rho}(x, 0) d x \\
& +\iint_{\operatorname{supp}\left|\frac{\partial^{2} \varphi_{\rho}}{\partial t^{2}}-\Delta \varphi_{\rho}\right|}|u|^{q} \varphi_{\rho} d x d t+\iint_{\varphi_{\rho}(x, t)=\xi(x)}|u|^{q} \xi(x) d x d t \\
& \leqslant \iint_{\operatorname{supp}\left|\frac{\partial^{2} \varphi_{\rho}}{\partial t^{2}}-\Delta \varphi_{\rho}\right|}|u|\left|\frac{\partial^{2} \varphi_{\rho}}{\partial t^{2}}-\Delta \varphi_{\rho}\right| d x d t \\
& \leqslant\left(\iint_{\operatorname{supp}\left|\frac{\partial^{2} \varphi_{\rho}}{\partial t^{2}}-\Delta \varphi_{\rho}\right|}|u|^{q} \varphi_{\rho} d x d t\right)^{1 / q} \\
& \times\left(\iint_{\operatorname{supp}\left|\frac{\partial^{2} \varphi_{\rho}}{\partial t^{2}}-\Delta \varphi_{\rho}\right|} \frac{\left|\frac{\partial^{2} \varphi_{\rho}}{\partial t^{2}}-\Delta \varphi_{\rho}\right|^{q^{\prime}}}{\varphi_{\rho}^{q^{\prime}}-1} d x d t\right)^{1 / q^{\prime}},
\end{aligned}
$$

откуда, отбрасывая неотрищательные начальные условия, с учетом оценки (1.9) (при $p=q^{\prime}$ ) для последнего интеграла справа, будем иметь

$$
\begin{aligned}
\iint_{\varphi_{\rho}(x, t)=\xi(x)}|u|^{q} \xi(x) d x d t & \leqslant \iint_{\operatorname{supp}\left|\frac{\partial^{2} \varphi_{\rho}}{\partial t^{2}}-\Delta \varphi_{\rho}\right|} \frac{\left|\frac{\partial^{2} \varphi_{\rho}}{\partial t^{2}}-\Delta \varphi_{\rho}\right|^{q^{\prime}}}{\varphi^{q^{\prime}-1}} d x d t \\
& \leqslant c_{0} \rho^{-2 q^{\prime}+s_{*}+N+1} .
\end{aligned}
$$

Так как подынтегральное выражение в левой части не зависит от $\rho$, можем перейти к пределу по $\rho \rightarrow \infty$. В случае

$$
-2 q^{\prime}+s_{*}+N+1 \leqslant 0
$$


это приводит к соотношению

$$
\int_{0}^{\infty} \int_{K}|u|^{q} \xi d x d t \leqslant c_{0}
$$

Тогда в силу абсолютной непрерывности интеграла Лебега по мере и ввиду оценки $\varphi_{\rho} \leqslant \xi$ имеем при $\rho \rightarrow \infty$

$$
\left.\iint_{\operatorname{supp}}\left|\frac{\partial^{2} \varphi_{\rho}}{\partial t^{2}}-\Delta \varphi_{\rho}\right|\right|^{q} \varphi_{\rho} d x d t \leqslant \iint_{\operatorname{supp}\left|\frac{\partial^{2} \varphi_{\rho}}{\partial t^{2}}-\Delta \varphi_{\rho}\right|}|u|^{q} \xi d x d t=\varepsilon(\rho) \rightarrow 0
$$

Возвращаясь теперь к неравенству (2.3), получим

$$
\iint_{\varphi_{\rho}(x, t)=\xi(x)}|u|^{q} \xi d x d t \leqslant \varepsilon^{1 / q}(\rho) c_{0}^{1 / q^{\prime}} \rightarrow 0
$$

при $\rho \rightarrow \infty$, т.е. в пределе

$$
\int_{0}^{\infty} \int_{K}|u|^{q} \xi d x d t=0
$$

Поскольку $\xi>0$ в $K$, то $u \equiv 0$, что противоречит предположению о сушествовании ненулевого решения (техника исследования критического случая $-2 q^{\prime}+$ $s_{*}+N+1=0$ заимствована из работы [15]).

Из неравенства

$$
-2 q^{\prime}+s_{*}+N+1 \leqslant 0
$$

получаем условие отсутствия нетривиального решения: $1<q \leqslant q^{*}$.

ЗАмЕЧАниЕ 2.2. Мы не налагаем требования неотрицательности решения, поскольку для гиперболических неравенств принцип максимума не имеет места и в общем случае говорить о положительных решениях нельзя.

ЗАМЕЧАНИЕ 2.3. Условие

$$
\left.\frac{\partial u}{\partial t}\right|_{t=0} \geqslant 0
$$

можно заменить интегральным вариантом

$$
\liminf _{\rho \rightarrow \infty} \int_{K \cap\{|x|<\rho\}} \frac{\partial u}{\partial t}(x, 0) \varphi_{\rho}(x, 0) d x \geqslant 0 .
$$

ЗАмечАниЕ 2.4. Требование существования суммируемого следа $\frac{\partial u}{\partial t}$ при $t=0$ и требование непрерывности функции $u(x, t)$ являются несколько искусственными и приводятся с целью упростить изложение. В действительности можно рассматривать решения из соответствуюших классов С. Л. Соболева и использовать известные свойства следов, или даже из класса $L_{q, \operatorname{loc}}(K \times(0, \infty))$, оговорив отдельно поведение решений в окрестности границы.

Из доказательства теоремы 2.1 легко получить априорную оценку решения в зависимости от параметра $\rho$. 
ТеОРема 2.2. Пусть $и(x, t)$ - решение задачи (2.1). Тогда при достаточно больших $\rho$ и при любом $q>1$ справедлива оценка

$$
\begin{aligned}
& \int_{K \cap\{|x|<\rho\}} \frac{\partial u}{\partial t}(x, 0) \xi(x) d x+\iint_{(K \times(0, \infty)) \cap\{|x|<\rho\} \cap\{t<\rho\}}|u|^{q} \xi d x d t \\
& \leqslant c_{1} \rho^{-2 q^{\prime}+s_{*}+N+1} .
\end{aligned}
$$

Интересно отметить, что эта теорема содержит также оценку начальных данных, что дает возможность при $q<q^{*}$ получить противоречие с предположением об отсутствии нетривиального решения для ненулевых начальных данных, не переходя к пределу по $\rho \rightarrow \infty$. Точнее, существует такое $\rho_{0}$, что при $\rho>\rho_{0}$ неравенство из теоремы 2.2 не может выполняться.

Действительно, оставляя в левой части неравенства из теоремы 2.2 только первое слагаемое, получаем

$$
\int_{K \cap\{|x|<\rho\}} \frac{\partial u}{\partial t}(x, 0) \xi(x) d x \leqslant c_{1} \rho^{-2 q^{\prime}+s_{*}+N+1} .
$$

Очевидно, если $-2 q^{\prime}+s^{*}+N+1<0$, то правая часть стремится к нулю при $\rho \rightarrow \infty$, а левая монотонно возрастает (точнее, не убывает, если начальные данные имеют компактный носитель). Таким образом, для любых начальных данных, начиная с некоторого $\rho_{0}$, приведенное неравенство перестает выполняться для $\rho>\rho_{0}$. Поскольку фактически неравенство рассматривается лишь на носителе функции $\varphi_{\rho}$, который лежит в полосе $0<t<2 \rho^{\theta} \equiv 2 \rho$, то из проведенного построения вытекает оценка времени существования локального по $t$ решения в зависимости от роста начальных данных (см. также работу автора [17], где аналогичные вопросы рассматриваются для неравенств в ограниченной по пространственным переменным $x$ области).

\section{§ 3. Неоднородная задача}

Приведем результат, аналогичный полученному в статье [21] для параболических уравнений на многообразиях. Введем в неравенство (2.1) дополнительное слагаемое $w(x) \geqslant 0, w(x) \in L_{1, \text { loc }}(K)$, т. е. рассмотрим задачу

$$
\begin{aligned}
\frac{\partial^{2} u}{\partial t^{2}}-\Delta u & \geqslant|u|^{q}+w(x) \quad \text { в } K \times(0, \infty), \quad w(x) \not \equiv 0, \\
\left.u\right|_{\partial K \times[0, \infty)} & \geqslant 0, \\
\left.\frac{\partial u}{\partial t}\right|_{t=0} & \geqslant 0, \quad u \neq \equiv 0 .
\end{aligned}
$$

Нас интересует вопрос о том, как повлияет введение неоднородности на критический показатель отсутствия решения. 
Слабое решение этой задачи понимается в смысле определения 2.1, т. е. предполагается выполненным интегральное неравенство

$$
\begin{aligned}
\int_{0}^{\infty} & \int_{\partial K} u \frac{\partial \varphi}{\partial n} d x d t+\int_{0}^{\infty} \int_{K} u\left(\frac{\partial^{2} \varphi}{\partial t^{2}}-\Delta \varphi\right) d x d t \\
\geqslant & \int_{0}^{\infty} \int_{K}|u|^{q} \varphi d x d t-\int_{K} u(x, 0) \frac{\partial \varphi}{\partial t}(x, 0) d x \\
& +\int_{K} \frac{\partial u}{\partial t}(x, 0) \varphi(x, 0) d x+\int_{0}^{\infty} \int_{K} w(x) \varphi d x d t
\end{aligned}
$$

TEOPEMA 3.1. Прu

$$
1<q<q^{*}=1+\frac{2}{s^{*}}
$$

задача (3.1) не имеет нетривиального глобального решения, каким бы малым ни было слагаемое $w(x) \geqslant 0, w(x) \not \equiv 0$.

ЗАмЕчАнИЕ 3.1. Интересно отметить, что критический показатель совпадает с соответствуюшим эллиптическим [22].

ДокАЗАТЕЛЬСтво. Следуя доказательству теоремы 2.1, получаем аналогичную теореме 2.2 оценку

$$
\begin{gathered}
\int_{0}^{\rho} \int_{K \cap\{|x|<\rho\}} w(x) \xi d x d t+\int_{K \cap\{|x|<\rho\}} \frac{\partial u}{\partial t}(x, 0) \xi(x) d x \\
\quad+\iint_{\varphi_{\rho}(x, t)=\xi(x)}|u|^{q} \xi d x d t \leqslant c_{1} \rho^{-2 q^{\prime}+s_{*}+N+1},
\end{gathered}
$$

откуда

$$
c_{1} \rho^{-2 q^{\prime}+s_{*}+N+1} \geqslant \int_{0}^{\rho} \int_{K \cap\{|x|<\rho\}} w(x) \xi(x) d x d t \geqslant \rho c_{w}
$$

при $\rho$ таких, что

$$
\int_{K \cap\{|x|<\rho\}} w(x) \xi(x) d x \geqslant c_{w} \equiv \text { const }>0 .
$$

Для получения противоречия при $\rho \rightarrow \infty$ достаточно предполагать, что $-2 q^{\prime}+$ $s_{*}+N<0$, т.е.

$$
q<1+\frac{2}{s^{*}}
$$

Теорема доказана. 


\section{$\S 4$. Сингулярные неравенства в конической области}

Пусть $R>0$ и $-2<\sigma<+\infty$. Рассмотрим проблему отсутствия слабых решений задачи

$$
\begin{aligned}
\frac{\partial^{2} u}{\partial t^{2}}-\Delta u & \geqslant|x|^{\sigma} u^{q} \quad \text { в } K_{R} \times(0, \infty), \\
\left.\frac{\partial u}{\partial t}\right|_{t=0} & \geqslant 0, \\
u & \geqslant 0, \quad u \neq 0 .
\end{aligned}
$$

В отличие от ранее приведенных результатов ограничимся изучением неотрицательных решений.

ОПРеДЕЛЕНИЕ 4.1. Пусть $u(x, t) \in C\left(\bar{K}_{R} \times[0, \infty)\right)$ и определен локально суммируемый след $\frac{\partial u}{\partial t}$ при $t=0$. Неотрицательная функция $u(x, t)$ называется слабъмм решением задачи (4.1), если для любой неотрицательной пробной функции $\varphi(x, t) \in W_{\infty}^{2}\left(K_{R} \times(0, \infty)\right)$ такой, что $\left.\varphi\right|_{\partial K_{R} \times(0, \infty)}=0$, и финитной по переменным $r=|x|$ и $t$, выполнено интегральное неравенство

$$
\begin{aligned}
& \int_{0}^{\infty} \int_{\partial K_{R}} u \frac{\partial \varphi}{\partial n} d x d t+\int_{0}^{\infty} \int_{K_{R}} u\left(\frac{\partial^{2} \varphi}{\partial t^{2}}-\Delta \varphi\right) d x d t \\
& \geqslant \int_{0}^{\infty} \int_{K_{R}}|x|^{\sigma} u^{q} \varphi d x d t-\int_{K_{R}} u(x, 0) \frac{\partial \varphi}{\partial t}(x, 0) d x \\
& \quad+\int_{K_{R}} \frac{\partial u}{\partial t}(x, 0) \varphi(x, 0) d x
\end{aligned}
$$

TeOpema 4.1. $\Pi p u$

$$
1<q \leqslant q^{*}=1+\frac{2+\sigma}{s^{*}+1}
$$

задача (4.1) не имеет нетривиального глобального решения.

ДокАЗАТЕЛьСтво. Пусть $u(x, t)$ - нетривиальное решение. Из неравенства $(4.2)$ с пробной функцией $\varphi(x, t)=\varphi_{\rho}(x, t)$, определенной в $(1.6)$, с $p=q^{\prime}>1$, $\theta=1$, имеем

$$
\begin{aligned}
& \int_{K_{R}} \frac{\partial u}{\partial t}(x, 0) \varphi_{\rho}(x, 0) d x+\int_{0}^{\infty} \int_{K_{R}} u^{q}|x|^{\sigma} \varphi_{\rho} d x d t \\
& \leqslant \int_{0}^{\infty} \int_{\partial K_{R}} u \frac{\partial \varphi_{\rho}}{\partial n} d x d t+\iint_{\frac{\partial^{2} \varphi_{\rho}}{\partial t^{2}}=0} u \frac{\partial^{2} \varphi_{\rho}}{\partial t^{2}} d x d t \\
& \quad-\iint_{\Delta \varphi_{\rho} \geqslant 0} u \Delta \varphi_{\rho} d x d t+\iint_{\frac{\partial^{2} \varphi_{\rho}}{\partial t^{2}} \neq 0} u \frac{\partial^{2} \varphi_{\rho}}{\partial t^{2}} d x d t \\
& \quad-\iint_{\Delta \varphi_{\rho}<0} u \Delta \varphi_{\rho} d x d t .
\end{aligned}
$$


Первый и третий интегралы в правой части неположительны, второй интеграл равен нулю. Применяя неравенство Гёльдера к оставшимся интегралам, получим

$$
\begin{aligned}
& \int_{K_{R}} \frac{\partial u}{\partial t}(x, 0) \varphi_{\rho}(x, 0) d x+\int_{0}^{\infty} \int_{K_{R}} u^{q}|x|^{\sigma} \varphi_{\rho} d x d t \\
& =\int_{K_{R}} \frac{\partial u}{\partial t}(x, 0) \varphi_{\rho}(x, 0) d x+\iint_{\varphi_{\rho}(x, t) \neq \xi(x)} u^{q}|x|^{\sigma} \varphi_{\rho} d x d t \\
& +\iint_{\varphi_{\rho}(x, t)=\xi(x)} u^{q}|x|^{\sigma} \xi(x) d x d t \\
& \leqslant \iint_{\operatorname{supp}\left|\frac{\partial^{2} \varphi_{\rho}}{\partial t^{2}}\right|} u\left|\frac{\partial^{2} \varphi_{\rho}}{\partial t^{2}}\right| d x d t+\iint_{\Delta \varphi_{\rho}<0} u\left|\Delta \varphi_{\rho}\right| d x d t \\
& \leqslant\left(\iint_{\operatorname{supp}\left|\frac{\partial^{2} \varphi_{\rho}}{\partial t^{2}}\right|} u^{q}|x|^{\sigma} \varphi_{\rho} d x d t\right)^{1 / q} \\
& \times\left(\iint_{\operatorname{supp}\left|\frac{\partial^{2} \varphi_{\rho}}{\partial t^{2}}\right|} \frac{\left|\frac{\partial^{2} \varphi_{\rho}}{\partial t^{2}}\right|^{q^{\prime}}}{\varphi_{\rho}^{q^{\prime}-1}|x|^{\sigma\left(q^{\prime}-1\right)}} d x d t\right)^{1 / q^{\prime}} \\
& +\left(\iint_{\Delta \varphi_{\rho}<0} u^{q}|x|^{\sigma} \varphi_{\rho} d x d t\right)^{1 / q} \\
& \times\left(\iint_{\Delta \varphi_{\rho}<0} \frac{\left|\Delta \varphi_{\rho}\right|^{q^{\prime}}}{\varphi_{\rho}^{q^{\prime}-1}|x|^{\sigma\left(q^{\prime}-1\right)}} d x d t\right)^{1 / q^{\prime}} .
\end{aligned}
$$

Наконец, используя неравенство Юнга с параметром, получаем

$$
\begin{aligned}
\iint_{\varphi_{\rho}(x, t)=\xi(x)} u^{q}|x|^{\sigma} \xi(x) d x d t \leqslant & c \iint_{\operatorname{supp}}\left|\frac{\partial^{2} \varphi_{\rho}}{\partial t^{2}}\right| \frac{\left|\frac{\partial^{2} \varphi_{\rho}}{\partial t^{2}}\right|^{q^{\prime}}}{\varphi_{\rho}^{q^{\prime}-1}|x|^{\sigma\left(q^{\prime}-1\right)}} d x d t \\
& +c \iint_{\Delta \varphi_{\rho}<0} \frac{\left|\Delta \varphi_{\rho}\right|^{q^{\prime}}}{\varphi_{\rho}^{q^{\prime}-1}|x|^{\sigma\left(q^{\prime}-1\right)}} d x d t
\end{aligned}
$$

Последнее слагаемое в силу неравенства (1.7) (c $p=q^{\prime}$ и $\left.\theta=1\right)$ не превосходит

$$
c \rho^{1-q^{\prime}(\sigma+2)+s_{*}+N+\sigma} .
$$

Если

$$
1-q^{\prime}(\sigma+2)+s_{*}+N+\sigma \leqslant 0,
$$

то последний интеграл в (4.5) ограничен при $\rho \rightarrow \infty$.

Легко видеть, что в условиях (4.6) первый интеграл в правой части (4.5) также ограничен. Действительно, если $s_{*}+N-\sigma\left(q^{\prime}-1\right)>0$, показатель роста в (1.8) совпадает с $1-q^{\prime}(\sigma+2)+s_{*}+N+\sigma \leqslant 0$. Если же $s_{*}+N-\sigma\left(q^{\prime}-1\right) \leqslant 0$, то множитель $\rho^{-\theta\left(2 q^{\prime}-1\right)}$ стремится к нулю при $\rho \rightarrow \infty$ и, следовательно, рассматриваемый интеграл ограничен. 
Переходя к пределу при $\rho \rightarrow \infty$, в случае (4.6) приходим к оценке

$$
\int_{0}^{\infty} \int_{K_{R}} u^{q}|x|^{\sigma} \xi d x d t \leqslant c_{0},
$$

откуда с помощью наших стандартных рассуждений получаем отсутствие нетривиального глобального решения при вьполнении неравенства (4.6). Теорема доказана.

Аналогичные результаты устанавливаются и для сингулярности по $t$. Ограничимся одним частным случаем. Пусть $T>0, R>0$ и $-2<\gamma \leqslant 0$. Рассмотрим проблему отсутствия слабых решений задачи

$$
\begin{aligned}
\frac{\partial^{2} u}{\partial t^{2}}-\Delta u & \geqslant t^{\gamma} u^{q} \quad \text { в } K_{R} \times(T, \infty), \\
\left.\frac{\partial u}{\partial t}\right|_{t=T} & \geqslant 0, \\
u & \geqslant 0, \quad u \neq 0 .
\end{aligned}
$$

ОПРЕДЕЛЕНИЕ 4.2. Пусть $u(x, t) \in C\left(\bar{K}_{R} \times[T, \infty)\right)$ и определен локально суммируемый след $\frac{\partial u}{\partial t}$ при $t=T$. Неотрищательная функция $u(x, t)$ называется $с л a-$ бым решением задачи (4.7), если для любой неотрищательной пробной функции $\varphi(x, t) \in W_{\infty}^{2}\left(K_{R} \times(T, \infty)\right)$, такой, что $\left.\varphi\right|_{\partial K_{R} \times(T, \infty)}=0$, и финитной по переменным $r=|x|$ и $t$, выполнено интегральное неравенство

$$
\begin{aligned}
\int_{T}^{\infty} & \int_{\partial K_{R}} u \frac{\partial \varphi}{\partial n} d x d t+\int_{T}^{\infty} \int_{K_{R}} u\left(\frac{\partial^{2} \varphi}{\partial t^{2}}-\Delta \varphi\right) d x d t \\
\geqslant & \int_{T}^{\infty} \int_{K_{R}} t^{\gamma} u^{q} \varphi d x d t-\int_{K_{R}} u(x, T) \frac{\partial \varphi}{\partial t}(x, T) d x \\
& +\int_{K_{R}} \frac{\partial u}{\partial t}(x, T) \varphi(x, T) d x .
\end{aligned}
$$

TEOPEMA 4.2. Прu $-2<\gamma \leqslant 0 u$

$$
1<q \leqslant q^{*}=1+\frac{2+\gamma}{s^{*}+1}
$$

задача (4.7) не имеет нетривиального глобального решения.

ДокАЗАтЕльство. Пусть $u(x, t)$ - нетривиальное решение. Действуя аналогично доказательству теоремы 4.1 (очевидно, можно брать ту же самую пробную функцию $\varphi_{\rho}$, в частности $\left.\theta=1\right)$, получаем

$$
\begin{aligned}
\iint_{\varphi_{\rho}(x, t)=\xi(x)} u^{q} t^{\gamma} \xi(x) d x d t \leqslant & c \iint_{\operatorname{supp}\left|\frac{\partial^{2} \varphi_{\rho}}{\partial t^{2}}\right|} \frac{\left|\frac{\partial^{2} \varphi_{\rho}}{\partial t^{2}}\right|^{q^{\prime}}}{\varphi_{\rho}^{q^{\prime}-1} t^{\gamma}\left(q^{\prime}-1\right)} d x d t \\
& +c \iint_{\Delta \varphi_{\rho}<0} \frac{\left|\Delta \varphi_{\rho}\right|^{q^{\prime}}}{\varphi_{\rho}^{q^{\prime}-1} t^{\gamma}\left(q^{\prime}-1\right)} d x d t
\end{aligned}
$$


Первое слагаемое в силу $(1.12)$ (с $\left.p=q^{\prime}\right)$ не превосходит

$$
c \rho^{-q^{\prime}(2+\gamma)+\gamma+s_{*}+N+1},
$$

т. е. интеграл ограничен при

$$
1-q^{\prime}(2+\gamma)+s_{*}+N+\gamma \leqslant 0 .
$$

В силу нашего предположения $\gamma \leqslant 0$ справедливо неравенство $1-\gamma\left(q^{\prime}-1\right)>0$, поэтому второй интеграл (по оценке (1.11)) также ограничен при условии (4.10).

Тогда, переходя к пределу при $\rho \rightarrow \infty$, приходим к оценке

$$
\int_{T}^{\infty} \int_{K_{R}} u^{q} t^{\gamma} \xi d x d t \leqslant c_{0},
$$

откуда получаем отсутствие нетривиального решения при выполнении неравенства (4.10). Теорема доказана.

\section{§5. Неравенство с переменными коэффициентами в главной части}

Рассмотрим проблему отсутствия неотрицательных слабых решений задачи

$$
\begin{aligned}
\frac{\partial^{2} u}{\partial t^{2}}-\operatorname{div}\left(|x|^{\alpha} D u\right) & \geqslant u^{q} \quad \text { в } K_{R} \times(0, \infty), \\
\left.\frac{\partial u}{\partial t}\right|_{t=0} & \geqslant 0, \\
u & \geqslant 0, \quad u \neq 0,
\end{aligned}
$$

где $-\infty<\alpha<2$. Далее слабое решение будет пониматься в следуюшем смысле.

ОПРедЕлЕниЕ 5.1. Пусть $u(x, t) \in C\left(\bar{K}_{R} \times[0, \infty)\right)$ и определен локально суммируемый след $\frac{\partial u}{\partial t}$ при $t=0$. Неотрицательная функция $u(x, t)$ называется $c л a-$ бъм решением задачи (5.1), если для любой неотрицательной пробной функции $\varphi(x, t) \in W_{\infty}^{2}\left(K_{R} \times(0, \infty)\right)$, такой, что $\left.\varphi\right|_{\partial K_{R} \times(0, \infty)}=0$, и финитной по переменным $r=|x|$ и $t$, выполнено интегральное неравенство

$$
\begin{aligned}
& \int_{0}^{\infty} \int_{\partial K_{R}} u|x|^{\alpha} \frac{\partial \varphi}{\partial n} d x d t+\int_{0}^{\infty} \int_{K_{R}} u\left(\frac{\partial^{2} \varphi}{\partial t^{2}}-\operatorname{div}\left(|x|^{\alpha} D \varphi\right)\right) d x d t \\
& \geqslant \int_{0}^{\infty} \int_{K_{R}} u^{q} \varphi d x d t-\int_{K_{R}} u(x, 0) \frac{\partial \varphi}{\partial t}(x, 0) d x+\int_{K_{R}} \frac{\partial u}{\partial t}(x, 0) \varphi(x, 0) d x
\end{aligned}
$$

Введем параметры

$$
\begin{gathered}
s_{\alpha}^{*}=\frac{\alpha+N-2}{2}+\sqrt{\left(\frac{\alpha+N-2}{2}\right)^{2}+\lambda_{\omega}}, \\
s_{* \alpha}=-\frac{\alpha+N-2}{2}+\sqrt{\left(\frac{\alpha+N-2}{2}\right)^{2}+\lambda_{\omega}} .
\end{gathered}
$$


TeOpema 5.1. Пусть $-\infty<\alpha<2$. Тогда прu

$$
1<q \leqslant q^{*}=1+\frac{2-\alpha}{s_{\alpha}^{*}+1-\alpha / 2}
$$

задача (5.1) не имеет нетривиального глобального решения.

ДоКАЗАТЕЛЬСТВО проведем от противного. Пусть $u(x, t)$ - нетривиальное решение задачи (5.1) при $1<q \leqslant q^{*}$. Выберем пробную функцию аналогично задаче (4.1), однако в качестве функции $\xi$ вместо (1.2) возьмем

$$
\xi_{\alpha}(x) \equiv \xi_{\alpha}(r, \omega)=\left(r^{s_{* \alpha}}-R^{s_{* \alpha}}\right) \Phi(\omega)
$$

Тогда функции $\psi_{\rho}(x)$ и $\varphi_{\rho}(x, t)$ принимают вид

$$
\psi_{\rho}(x)=\xi_{\alpha}(x) \eta\left(\frac{|x|}{\rho}\right), \quad \varphi_{\rho}(x, t)=\psi_{\rho}(x) \eta\left(\frac{t}{\rho^{\theta}}\right) .
$$

В этом случае вместо оператора Лапласа мы имеем оператор

$$
A_{\alpha} \equiv \operatorname{div}\left(|x|^{\alpha} D\right)=r^{\alpha}\left(\frac{\partial^{2}}{\partial r^{2}}+\frac{\alpha+N-1}{r} \frac{\partial}{\partial r}+\frac{1}{r^{2}} \Delta_{\omega}\right)
$$

Легко убедиться в том, что

$$
\begin{aligned}
A_{\alpha} \xi_{\alpha} & \equiv \operatorname{div}\left(|x|^{\alpha} D \xi_{\alpha}\right)=\operatorname{div}\left(|x|^{\alpha} r^{s_{* \alpha} \Phi}\right)-R^{s_{* \alpha}} \operatorname{div}\left(|x|^{\alpha} \Phi\right) \\
& =r^{\alpha} r^{s_{* \alpha}-2}\left(s_{* \alpha}\left(s_{* \alpha}-1\right)+s_{* \alpha}(\alpha+N-1)-\lambda_{\omega}\right) \Phi(\omega)-R^{s_{* \alpha}} \frac{r^{\alpha}}{r^{2}} \Delta_{\omega} \Phi \\
& \equiv 0+\lambda_{\omega} \frac{R^{s_{*}}}{r^{2-\alpha}} \Phi(\omega) \geqslant 0
\end{aligned}
$$

в области $K_{R}$. Также $\left.\xi_{\alpha}\right|_{\partial K_{R}}=0$ и $\left.\frac{\partial \xi_{\alpha}}{\partial n}\right|_{\partial K_{R}} \leqslant 0$.

Можно получить аналоги оценок (1.7) и (1.8) для оператора $A_{\alpha}$ :

$$
\begin{aligned}
& \iint_{\left\{x, t: A_{\alpha} \varphi_{\rho}(x)<0\right\}} \frac{\left|A_{\alpha} \varphi_{\rho}(x, t)\right|^{p}}{\varphi_{\rho}^{p-1}(x, t)} d x d t \\
& \leqslant \int_{0}^{2 \rho^{\theta}} \eta\left(\frac{t}{\rho^{\theta}}\right) d t \int_{\left\{x: A_{\alpha} \psi_{\rho}(x)<0\right\}} \frac{\left|A_{\alpha} \psi_{\rho}\right|^{p}}{\psi_{\rho}^{p-1}} d x \leqslant c \rho^{\theta-(2-\alpha) p+s_{* \alpha}+N}, \\
& \iint_{\operatorname{supp}}\left|\frac{\partial^{2} \varphi_{\rho}}{\partial t^{2}}\right| \frac{\left|\frac{\partial^{2} \varphi_{\rho}(x, t)}{\partial t^{2}}\right|^{p}}{\varphi_{\rho}^{p-1}(x, t)} d x d t \\
& \leqslant \int_{K_{R} \cap\{r<2 \rho\}} \psi_{\rho}(x) d x \int_{\operatorname{supp}\left|\frac{d^{2} \eta\left(t / \rho^{\theta}\right)}{d t^{2}}\right|} \frac{\left|\frac{d^{2} \eta\left(t / \rho^{\theta}\right)}{d t^{2}}\right|^{p}}{\eta^{p-1}\left(t / \rho^{\theta}\right)} d t \leqslant c \rho^{s_{* \alpha}+N-\theta(2 p-1)} .
\end{aligned}
$$


При $\theta=1-\alpha / 2>0$ степени в правых частях оценок совпадают и равны

$$
-(2-\alpha) p+s_{* \alpha}+N+1-\frac{\alpha}{2} .
$$

Далее действуем по схеме доказательства теоремы 4.1. Из определения слабого решения (с учетом равенства $\left.\frac{\partial \varphi_{\rho}}{\partial t}(x, 0)=0\right)$ имеем

$$
\begin{aligned}
& \int_{K_{R}} \frac{\partial u}{\partial t}(x, 0) \psi_{\rho}(x) d x+\int_{0}^{\infty} \int_{K_{R}} u^{q} \varphi_{\rho} d x d t \\
& \leqslant \int_{0}^{\infty} \int_{\partial K_{R}} u|x|^{\alpha} \frac{\partial \varphi_{\rho}}{\partial n} d x d t+\iint_{\operatorname{supp}\left|\frac{\partial^{2} \varphi_{\rho}}{\partial t^{2}}\right|} u\left|\frac{\partial^{2} \varphi_{\rho}}{\partial t^{2}}\right| d x d t \\
& \quad+\iint_{\left\{x, t: A_{\alpha} \varphi_{\rho}<0\right\}} u\left|A_{\alpha} \varphi_{\rho}\right| d x d t
\end{aligned}
$$

откуда (поскольку первый интеграл в правой части неположителен) после применения неравенства Гёльдера получаем

$$
\begin{aligned}
& \iint_{\varphi_{\rho}(x, t)=\xi_{\alpha}(x)} u^{q} \xi_{\alpha} d x d t \leqslant \iint_{\operatorname{supp}\left|\frac{\partial^{2} \varphi_{\rho}}{\partial t^{2}}\right|} \frac{\left|\frac{\partial^{2} \varphi_{\rho}}{\partial t^{2}}\right|^{q^{\prime}}}{\varphi_{\rho}^{q^{\prime}-1}} d x d t \\
& \quad+\iint_{\left\{x, t: A_{\alpha} \varphi_{\rho}<0\right\}} \frac{\left|A_{\alpha} \varphi_{\rho}\right|^{q^{\prime}}}{\varphi_{\rho}^{q^{\prime}-1}} d x d t \leqslant c_{0} \rho^{-(2-\alpha) q^{\prime}+s_{* \alpha}+N+1-\alpha / 2},
\end{aligned}
$$

что дает условие отсутствия нетривиального решения

$$
-(2-\alpha) q^{\prime}+s_{* \alpha}+N+1-\frac{\alpha}{2} \leqslant 0
$$

Отсюда $q \leqslant 1+\frac{2-\alpha}{s_{* \alpha}+N-1+\alpha / 2}$. Так как $N+s_{* \alpha}=s_{\alpha}^{*}+2-\alpha$, то приходим к утверждению теоремы.

\section{§6. Система неравенств}

Рассмотрим слабо связанную систему

$$
\begin{aligned}
\frac{\partial^{2} u}{\partial t^{2}}-\Delta u & \geqslant|v|^{q_{1}} \quad \text { в } K \times(0, \infty), \\
\frac{\partial^{2} v}{\partial t^{2}}-\Delta v & \geqslant|u|^{q_{2}} \quad \text { в } K \times(0, \infty), \\
\left.u\right|_{\partial K \times[0, \infty)} & \geqslant 0,\left.\quad v\right|_{\partial K \times[0, \infty)} \geqslant 0, \\
\left.\frac{\partial u}{\partial t}\right|_{t=0} & \geqslant 0,\left.\quad \frac{\partial v}{\partial t}\right|_{t=0} \geqslant 0, \quad u \neq 0, \quad v \neq 0 .
\end{aligned}
$$

ОПРЕДЕЛЕНИЕ 6.1 . Пара функций $u, v \in C(\bar{K} \times[0, \infty))$, у которых определены локально суммируемые следы $\frac{\partial u}{\partial t}$ и $\frac{\partial v}{\partial t}$ при $t=0$, называется слабым решением задачи (6.1), если для любой неотрицательной пробной функции $\varphi(x, t) \in W_{\infty}^{2}(K \times$ 
$(0, \infty))$, такой, что $\left.\varphi\right|_{\partial K \times(0, \infty)}=0$, и финитной по переменным $r=|x|$ и $t$, выполнены интегральные неравенства

$$
\begin{aligned}
\int_{0}^{\infty} \int_{\partial K} u \frac{\partial \varphi}{\partial n} d x d t+\int_{0}^{\infty} \int_{K} u\left(\frac{\partial^{2} \varphi}{\partial t^{2}}-\Delta \varphi\right) d x d t \\
\quad \geqslant \int_{0}^{\infty} \int_{K}|v|^{q_{1}} \varphi d x d t-\int_{K} u(x, 0) \frac{\partial \varphi}{\partial t}(x, 0) d x+\int_{K} \frac{\partial u}{\partial t}(x, 0) \varphi(x, 0) d x \\
\int_{0}^{\infty} \int_{\partial K} v \frac{\partial \varphi}{\partial n} d x d t+\int_{0}^{\infty} \int_{K} v\left(\frac{\partial^{2} \varphi}{\partial t^{2}}-\Delta \varphi\right) d x d t \\
\quad \geqslant \int_{0}^{\infty} \int_{K}|u|^{q_{2}} \varphi d x d t-\int_{K} v(x, 0) \frac{\partial \varphi}{\partial t}(x, 0) d x+\int_{K} \frac{\partial v}{\partial t}(x, 0) \varphi(x, 0) d x
\end{aligned}
$$

Теорема 6.1. Задача (6.1) не имеет слабого решения, если

$$
\max \left\{\gamma_{1}, \gamma_{2}\right\} \geqslant \frac{s^{*}+1}{2}, \quad \text { əде } \quad \gamma_{1}=\frac{q_{1}+1}{q_{1} q_{2}-1}, \quad \gamma_{2}=\frac{q_{2}+1}{q_{1} q_{2}-1}, \quad q_{1}>1, \quad q_{2}>1
$$

$s^{*}$ определяется формулой (1.1).

ЗАмечАниЕ 6.1. Для всего пространства $\mathbb{R}^{N}$ получаем (полагая $s^{*}=N-2$ ) известные условия отсутствия решения [13] (см. также [10])

$$
\max \left\{\frac{q_{1}+1}{q_{1} q_{2}-1}, \frac{q_{2}+1}{q_{1} q_{2}-1}\right\} \geqslant \frac{N-1}{2} .
$$

ДокАЗАТЕЛЬСТво. Пусть $u, v$ - слабое решение задачи (6.1). Выберем пробную функцию, как в доказательстве теоремы 2.1 , и будем использовать соответствующие обозначения. В частности, справедлива оценка (1.9).

Применяя в определении слабого решения неравенство Гёльдера, приходим к неравенствам

$$
\begin{aligned}
\int_{K} & \frac{\partial u}{\partial t}(x, 0) \varphi_{\rho}(x, 0) d x-\int_{K} u(x, 0) \frac{\partial \varphi_{\rho}}{\partial t}(x, 0) d x+\int_{0}^{\infty} \int_{K}|v|^{q_{1}} \varphi_{\rho} d x d t \\
\leqslant & \left(\iint_{\operatorname{supp}\left|\frac{\partial^{2} \varphi_{\rho}}{\partial t^{2}}-\Delta \varphi_{\rho}\right|}|u|^{q_{2}} \varphi_{\rho} d x d t\right)^{1 / q_{2}} \\
& \times\left(\iint_{\operatorname{supp}\left|\frac{\partial^{2} \varphi_{\rho}}{\partial t^{2}}-\Delta \varphi_{\rho}\right|} \frac{\left|\frac{\partial^{2} \varphi_{\rho}}{\partial t^{2}}-\Delta \varphi_{\rho}\right|^{q_{2}^{\prime}}}{\varphi_{\rho}^{q_{2}^{\prime}}-1} d x d t\right)^{1 / q_{2}^{\prime}} \\
\equiv & \left(\iint_{\operatorname{supp}\left|\frac{\partial^{2} \varphi_{\rho}}{\partial t^{2}}-\Delta \varphi_{\rho}\right|}|u|^{q_{2}} \varphi_{\rho} d x d t\right)^{1 / q_{2}} J_{1}^{1 / q_{2}^{\prime}}
\end{aligned}
$$




$$
\begin{aligned}
\int_{K} \frac{\partial v}{\partial t}(x, 0) \varphi_{\rho}(x, 0) d x-\int_{K} v(x, 0) \frac{\partial \varphi_{\rho}}{\partial t}(x, 0) d x+\int_{0}^{\infty} \int_{K}|u|^{q_{2}} \varphi_{\rho} d x d t \\
\leqslant\left(\iint_{\operatorname{supp}\left|\frac{\partial^{2} \varphi_{\rho}}{\partial t^{2}}-\Delta \varphi_{\rho}\right|}|v|^{q_{1}} \varphi_{\rho} d x d t\right)^{1 / q_{1}} \\
\quad \times\left(\iint_{\operatorname{supp}\left|\frac{\partial^{2} \varphi_{\rho}}{\partial t^{2}}-\Delta \varphi_{\rho}\right|} \frac{\left|\frac{\partial^{2} \varphi_{\rho}}{\partial t^{2}}-\Delta \varphi_{\rho}\right|^{q_{1}^{\prime}}}{\varphi_{\rho}^{q_{1}^{\prime}-1}} d x d t\right)^{1 / q_{1}^{\prime}} \\
\equiv\left(\iint_{\operatorname{supp}\left|\frac{\partial^{2} \varphi_{\rho}}{\partial t^{2}}-\Delta \varphi_{\rho}\right|}|v|^{q_{1}} \varphi_{\rho} d x d t\right)^{1 / q_{1}} J_{2}^{1 / q_{1}^{\prime}},
\end{aligned}
$$

причем согласно (1.9)

$$
J_{1} \leqslant c_{0} \rho^{-2 q_{2}^{\prime}+s_{*}+N+1}, \quad J_{2} \leqslant c_{0} \rho^{-2 q_{1}^{\prime}+s_{*}+N+1} .
$$

Подставим неравенство (6.3) в (6.2). Тогда

$$
\begin{aligned}
\int_{K} \frac{\partial u}{\partial t} & (x, 0) \psi_{\rho}(x) d x+\int_{0}^{\infty} \int_{K}|v|^{q_{1}} \varphi_{\rho} d x d t \\
& \leqslant\left(\iint_{\operatorname{supp}\left|\frac{\partial^{2} \varphi_{\rho}}{\partial t^{2}}-\Delta \varphi_{\rho}\right|}|v|^{q_{1}} \varphi_{\rho} d x d t\right)^{1 /\left(q_{1} q_{2}\right)} J_{2}^{1 /\left(q_{1}^{\prime} q_{2}\right)} J_{1}^{1 / q_{2}^{\prime}}
\end{aligned}
$$

откуда после упрощения степеней получаем

$$
\begin{aligned}
& \int_{0}^{\infty} \int_{K}|v|^{q_{1}} \varphi_{\rho} d x d t \leqslant\left(J_{2}^{q_{1}-1} J_{1}^{q_{1}\left(q_{2}-1\right)}\right)^{1 /\left(q_{1} q_{2}-1\right)} \\
& \quad \leqslant C \rho^{\left(\left(s_{*}+N-2+1\right)\left(q_{1} q_{2}-1\right)-2\left(q_{1}+1\right)\right) /\left(q_{1} q_{2}-1\right)}=C \rho^{s^{*}+1-2 \gamma_{1}} .
\end{aligned}
$$

Отсюда, рассуждая, как и ранее, выводим отсутствие нетривиального решения $v(x, t)$ в случае

$$
s^{*}+1-2 \gamma_{1} \leqslant 0 .
$$

Аналогично, подставляя неравенство (6.2) в (6.3), приходим к оценке

$$
\begin{aligned}
& \int_{0}^{\infty} \int_{K}|u|^{q_{2}} \varphi_{\rho} d x d t \leqslant\left(J_{1}^{q_{2}-1} J_{2}^{q_{2}\left(q_{1}-1\right)}\right)^{1 /\left(q_{1} q_{2}-1\right)} \\
& \quad \leqslant C \rho^{\left(\left(s_{*}+N-2+1\right)\left(q_{1} q_{2}-1\right)-2\left(q_{2}+1\right)\right) /\left(q_{1} q_{2}-1\right)}=C \rho^{s^{*}+1-2 \gamma_{2}},
\end{aligned}
$$

т. е. нетривиальное решение $u(x, t)$ отсутствует при $s^{*}+1-2 \gamma_{2} \leqslant 0$.

Очевидно, что если хотя бы одна из функций $u(x, t)$ или $v(x, t)$ тождественно равна нулю, то равна нулю и другая. Таким образом, условие отсутствия нетривиального решения принимает вид

$$
\max \left\{\gamma_{1}, \gamma_{2}\right\} \geqslant \frac{s^{*}+1}{2} .
$$


Теорема доказана.

Система неоднородных неравенств

$$
\begin{aligned}
\frac{\partial^{2} u}{\partial t^{2}}-\Delta u & \geqslant|v|^{q_{1}}+w_{1}(x) \quad \text { в } K \times(0, \infty), \\
\frac{\partial^{2} v}{\partial t^{2}}-\Delta v & \geqslant|u|^{q_{2}}+w_{2}(x) \quad \text { в } K \times(0, \infty), \\
\left.u\right|_{\partial K \times[0, \infty)} & \geqslant 0,\left.\quad v\right|_{\partial K \times[0, \infty)} \geqslant 0, \\
\left.\frac{\partial u}{\partial t}\right|_{t=0} & \geqslant 0,\left.\quad \frac{\partial v}{\partial t}\right|_{t=0} \geqslant 0, \quad u \neq 0, \quad v \neq 0,
\end{aligned}
$$

исследуется аналогично. Оценка (6.4) в этом случае (в предположениях теоремы 3.1) принимает вид

$$
c_{w} \rho \leqslant \int_{0}^{\rho} \int_{K} w_{1}(x) d x d t+\int_{0}^{\infty} \int_{K}|v|^{q_{1}} \varphi_{\rho} d x d t \leqslant C \rho^{s^{*}+1-2 \gamma_{1}}
$$

Выполнение этого неравенства при $s^{*}-2 \gamma_{1}<0$, очевидно, невозможно. Из неравенства (6.5) получим

$$
c_{w} \rho \leqslant \int_{0}^{\rho} \int_{K} w_{2}(x) d x d t+\int_{0}^{\infty} \int_{K}|u|^{q_{2}} \varphi_{\rho} d x d t \leqslant C \rho^{s^{*}+1-2 \gamma_{2}}
$$

т. е. при $s^{*}-2 \gamma_{1}<0$ решения нет.

Объединяя полученные неравенства, приходим к соответствующему утверждению.

ТЕОРЕма 6.2. Задача (6.6) не имеет глобального нетривиального решения, если

$$
\max \left\{\gamma_{1}, \gamma_{2}\right\}>\frac{s^{*}}{2}, \quad \text { əде } \quad \gamma_{1}=\frac{q_{1}+1}{q_{1} q_{2}-1}, \quad \gamma_{2}=\frac{q_{2}+1}{q_{1} q_{2}-1}, \quad q_{1}>1, \quad q_{2}>1
$$

$s^{*}$ определяется формулой (1.1), какими бы малыми ни были локально суммируемые неотрицательные функиии $w_{1}(x) \not \equiv 0 u w_{2}(x) \not \equiv 0$.

ЗАмЕчАниЕ 6.2. Следуя предлагаемому методу, возможно исследовать зависимость критических показателей от роста функций $w_{1}(x)$ и $w_{2}(x)$ при $|x| \rightarrow \infty$, а также рассмотреть локально суммируемые неоднородности общего вида $w_{1}(x, t)$ и $w_{2}(x, t)$. 


\section{§ 7. Эволюционные неравенства и системы высокого порядка по переменной $t$}

Пусть $k \in \mathbb{N}$. Рассмотрим проблему отсутствия слабых решений задачи

$$
\begin{aligned}
\frac{\partial^{k} u}{\partial t^{k}}-\Delta u & \geqslant|u|^{q} \quad \text { в } K \times(0, \infty), \\
\left.u\right|_{\partial K \times[0, \infty)} & \geqslant 0, \\
\left.\frac{\partial^{k-1} u}{\partial t^{k-1}}\right|_{t=0} & \geqslant 0, \quad u \neq 0 .
\end{aligned}
$$

При $k=1$ имеем параболическую задачу, при $k=2$ - гиперболическую, а при $k \geqslant 3$ будем называть такую задачу әволюционным неравенством высокого порядка по переменной $t$, поскольку в этом случае она не имеет определенного типа. Последнее обстоятельство не позволяет применять к ее исследованию стандартные методы линейной теории, которая для такого класса задач еще не разработана.

В то же время с помощью метода пробных функций получить результаты об отсутствии глобальных нетривиальных решений можно для всех $k \in \mathbb{N}$. Мы, однако, не рассматриваем вопрос о неулучшаемости этих условий для $k>2$. Отсутствие решений для аналогичных задач в ограниченной (по $x$ ) области исследовано в работе автора [17].

Не останавливаясь на деталях, отметим, что мы будем использовать пробные функции из анизотропных пространств С. Л. Соболева $W_{\infty}^{2, k}(K \times(0, \infty))$.

ОПРЕДЕЛЕНИЕ 7.1. Пусть $u(x, t) \in C(\bar{K} \times[0, \infty))$ и определены локально суммируемые следы $\frac{\partial^{i} u}{\partial t^{i}}$ при $t=0$ для $i=1, \ldots, k-1$. Функция $u(x, t)$ называется $c л a-$ бым решением задачи (7.1), если для любой неотрицательной пробной функции $\varphi(x, t) \in W_{\infty}^{2, k}(K \times(0, \infty))$, такой, что $\left.\varphi\right|_{\partial K \times(0, \infty)}=0$, и финитной по переменным $r=|x|$ и $t$, выполнено интегральное неравенство

$$
\begin{aligned}
\int_{0}^{\infty} \int_{\partial K} u \frac{\partial \varphi}{\partial n} d x d t+\int_{0}^{\infty} \int_{K} u\left((-1)^{k} \frac{\partial^{k} \varphi}{\partial t^{k}}-\Delta \varphi\right) d x d t \\
\geqslant \int_{0}^{\infty} \int_{K}|u|^{q} \varphi d x d t+\sum_{i=1}^{k-1}(-1)^{i} \int_{K} \frac{\partial^{k-1-i} u}{\partial t^{k-1-i}}(x, 0) \frac{\partial^{i} \varphi}{\partial t^{i}}(x, 0) d x \\
\quad+\int_{K} \frac{\partial^{k-1} u}{\partial t^{k-1}}(x, 0) \varphi(x, 0) d x .
\end{aligned}
$$

TeOpema 7.1. Пpu

$$
1<q \leqslant q^{*}=1+\frac{2}{s^{*}+2 / k}
$$

задача (7.1) не имеет нетривиального глобального решения. 
ДокАЗАТЕЛЬСТво. Действуя аналогично предыдушим построениям пробной функции, рассмотрим функцию

$$
\eta(y)=(\zeta(y))^{k p_{0}}
$$

с некоторым положительным параметром $p_{0}$. Тогда явным образом можно проверить выполнение оценки (для $1<p \leqslant p_{0}$ )

$$
\left|\eta^{(k)}(y)\right|^{p}=c_{\eta} \eta^{p-1}(y)
$$

с некоторой постоянной $c_{\eta}$.

Вводя замену переменной $y=t / \rho^{\theta}$, приходим к обобшению неравенства (1.3):

$$
\int_{\operatorname{supp}}\left|\frac{d^{k} \eta\left(t / \rho^{\theta}\right)}{d t^{k}}\right| \frac{\left|\frac{d^{k} \eta\left(t / \rho^{\theta}\right)}{d t^{k}}\right|^{p}}{\eta^{p-1}\left(t / \rho^{\theta}\right)} d t \leqslant c_{\eta} \rho^{-\theta(k p-1)}
$$

откуда для пробной функции $\varphi_{\rho}(x, t)$, введенной по формуле $(1.6)$ с $\eta$, определенной в (7.2), выводим оценку

$$
\iint_{\operatorname{supp}}\left|\frac{\partial^{k} \varphi_{\rho}}{\partial t^{k}}\right| \frac{\left|\frac{\partial^{k} \varphi_{\rho}(x, t)}{\partial t^{k}}\right|^{p}}{\varphi_{\rho}^{p-1}(x, t)} d x d t \leqslant \rho^{s_{*}+N-\theta(k p-1)}
$$

Тогда, поскольку в оценке (1.7) для $\sigma=0$ ничего не меняется, мы выбираем $\theta$ из условия равенства степеней

$$
s_{*}+N-\theta(k p-1)=\theta-2 p+s_{*}+N,
$$

T. e.

$$
\theta=\frac{2}{k}
$$

Окончательно приходим к оценке

$$
\iint_{\operatorname{supp}\left|(-1)^{k} \frac{\partial^{k} \varphi_{\rho}}{\partial t^{k}}-\Delta \varphi_{\rho}\right|} \frac{\left|(-1)^{k} \frac{\partial^{k} \varphi_{\rho}}{\partial t^{k}}-\Delta \varphi_{\rho}\right|^{p}}{\varphi_{\rho}^{p-1}} d x d t \leqslant c_{0} \rho^{-2 p+s_{*}+N+2 / k} .
$$

Пусть $u(x, t)$ - нетривиальное решение задачи (7.1) с $1<q \leqslant q^{*}$. По определению для пробной функции $\varphi(x, t)=\varphi_{\rho}(x, t)$ с учетом очевидных равенств

$$
\frac{\partial^{i} \varphi_{\rho}}{\partial t^{i}}(x, 0) \equiv 0, \quad i=1, \ldots, k-1,
$$


это означает, что

$$
\begin{aligned}
& \int_{K} \frac{\partial^{k-1} u}{\partial t^{k-1}}(x, 0) \varphi_{\rho}(x, 0) d x+\int_{0}^{\infty} \int_{K}|u|^{q} \varphi_{\rho} d x d t \leqslant \int_{0}^{\infty} \int_{\partial K} u \frac{\partial \varphi_{\rho}}{\partial n} d x d t \\
& \quad+\iint_{\operatorname{supp}\left|(-1)^{k} \frac{\partial^{k} \varphi_{\rho}}{\partial t^{k}}-\Delta \varphi_{\rho}\right|} u\left((-1)^{k} \frac{\partial^{k} \varphi_{\rho}}{\partial t^{k}}-\Delta \varphi_{\rho}\right) d x d t
\end{aligned}
$$

Отсюда, действуя согласно использованному ранее методу, с учетом оценки (7.5) при $p=q^{\prime}$ приходим к отсутствию нетривиального глобального решения при выполнении условия

$$
-2 q^{\prime}+s_{*}+N+\frac{2}{k} \leqslant 0
$$

т.e.

$$
q \leqslant 1+\frac{2}{s^{*}+2 / k}
$$

ЗАмЕЧАНИЕ 7.1. Частным случаем теоремы 7.1 при $k=1$ является утверждение об отсутствии нетривиального глобального решения параболической задачи

$$
\begin{aligned}
\frac{\partial u}{\partial t}-\Delta u & \geqslant|u|^{q} \quad \text { в } K \times(0, \infty), \\
\left.u\right|_{\partial K \times[0, \infty)} & \geqslant 0, \\
\left.u\right|_{t=0} & \geqslant 0, \quad u \neq 0 .
\end{aligned}
$$

Сформулируем соответствуюший результат.

TEOPEMA 7.2. Прu

$$
1<q \leqslant 1+\frac{2}{s^{*}+2}
$$

задача (7.7) не имеет нетривиального глобального решения.

ЗАмЕчАнИЕ 7.2. Для всего $\mathbb{R}^{N}$ (при $s^{*}=N-2$ ) теорема 7.1 утверждает, что задача

$$
\begin{aligned}
& \frac{\partial^{k} u}{\partial t^{k}}-\Delta u \geqslant|u|^{q} \quad \text { в } \mathbb{R}^{N} \times(0, \infty), \\
& \left.\frac{\partial^{k-1} u}{\partial t^{k-1}}\right|_{t=0} \geqslant 0, \quad u \neq 0,
\end{aligned}
$$

не имеет глобального нетривиального решения, если

$$
1<q \leqslant 1+\frac{2}{N-2+2 / k}
$$

Для параболического случая $k=1$ приходим к критическому показателю Фужиты $q^{*}=1+2 / N$, для гиперболического $k=2-$ к критическому показателю Като $q^{*}=\frac{N+1}{N-1}$. 
Аналогичные результаты можно установить и для систем вида

$$
\begin{aligned}
& \frac{\partial^{k} u}{\partial t^{k}}-\Delta u \geqslant|v|^{q_{1}} \quad \text { в } K \times(0, \infty), \\
& \frac{\partial^{k} v}{\partial t^{k}}-\Delta v \geqslant|u|^{q_{2}} \quad \text { в } K \times(0, \infty), \\
&\left.u\right|_{\partial K \times[0, \infty)} \geqslant 0,\left.\quad v\right|_{\partial K \times[0, \infty)} \geqslant 0, \\
&\left.\frac{\partial^{k-1} u}{\partial t^{k-1}}\right|_{t=0} \geqslant 0,\left.\quad \frac{\partial^{k-1} v}{\partial t^{k-1}}\right|_{t=0} \geqslant 0, \quad u \neq 0, \quad v \neq 0 .
\end{aligned}
$$

ТЕОРЕМА 7.3. Задача (7.8) не имеет глобального нетривиального решения (понимаемого в смысле определений 7.1 и 6.1), если

$2 \partial e$

$$
\max \left\{\gamma_{1}, \gamma_{2}\right\} \geqslant \frac{s^{*}+2 / k}{2}
$$

$$
\gamma_{1}=\frac{q_{1}+1}{q_{1} q_{2}-1}, \quad \gamma_{2}=\frac{q_{2}+1}{q_{1} q_{2}-1}, \quad q_{1}>1, \quad q_{2}>1
$$

$s^{*}$ определяется формулой (1.1).

ДокАЗАТЕЛЬСтво. Следуя доказательствам теорем 6.1 и 7.1, получаем оценки интегралов

$$
J(p) \equiv \iint_{\operatorname{supp}\left|(-1)^{k} \frac{\partial^{k} \varphi_{\rho}}{\partial t^{k}}-\Delta \varphi_{\rho}\right|} \frac{\left|(-1)^{k} \frac{\partial^{k} \varphi_{\rho}}{\partial t^{k}}-\Delta \varphi_{\rho}\right|^{p}}{\varphi_{\rho}^{p-1}} d x d t \leqslant c_{0} \rho^{-2 p+s_{*}+N+2 / k}
$$

T. e.

$$
J_{1} \equiv J\left(q_{2}\right) \leqslant c_{0} \rho^{-2 q_{2}^{\prime}+s_{*}+N+2 / k}, \quad J_{2} \equiv J\left(q_{1}\right) \leqslant c_{0} \rho^{-2 q_{1}^{\prime}+s_{*}+N+2 / k} .
$$

Тогда можно установить оценки

$$
\begin{aligned}
& \int_{0}^{\infty} \int_{K}|v|^{q_{1}} \varphi_{\rho} d x d t \leqslant\left(J_{2}^{q_{1}-1} J_{1}^{q_{1}\left(q_{2}-1\right)}\right)^{1 /\left(q_{1} q_{2}-1\right)} \\
& \leqslant C \rho^{\left(\left(s_{*}+N-2+2 / k\right)\left(q_{1} q_{2}-1\right)-2\left(q_{1}+1\right)\right) /\left(q_{1} q_{2}-1\right)}=C \rho^{s^{*}+2 / k-2 \gamma_{1}}, \\
& \int_{0}^{\infty} \int_{K}|u|^{q_{2}} \varphi_{\rho} d x d t \leqslant\left(J_{1}^{q_{2}-1} J_{2}^{q_{2}\left(q_{1}-1\right)}\right)^{1 /\left(q_{1} q_{2}-1\right)} \\
& \leqslant C \rho^{\left(\left(s_{*}+N-2+2 / k\right)\left(q_{1} q_{2}-1\right)-2\left(q_{2}+1\right)\right) /\left(q_{1} q_{2}-1\right)}=C \rho^{s^{*}+2 / k-2 \gamma_{2}},
\end{aligned}
$$

откуда получаем требуемое условие отсутствия нетривиальных решений

$$
\max \left\{\gamma_{1}, \gamma_{2}\right\} \geqslant \frac{s^{*}+2 / k}{2} .
$$

В заключение автор выражает благодарность С. И. Похожаеву за постановку задачи и обсуждение результатов.

\section{Список литературы}

1. Самарский А.А., Галактионов В.А., Курдюмов С. П., Михайлов А.П. Режимыс обострением в задачах для квазилинейных параболических уравнений. М.: Наука, 1987.

2. Alinhac S. Blow-up for nonlinear hyperbolic equations. Birkhäuser: Boston, 1995.

3. Georgiev V., Linblad H., Sogge C. Weighted Strichartz estimate and global existence for semilinear wave equation // Amer. J. Math. 1997. V. 119. P. 1291-1319. 
4. Galaktionov V. A., Pohozaev S. I. Blow-up, critical exponents and asymptotic spectra for nonlinear hyperbolic equations. Math. Preprint Univ. of Bath 00/10, 2000.

5. Коньков A. A. О неотрицательных решениях квазилинейных эллиптических неравенств // Изв. РАН. Сер. матем. 1999. Т. 63. № 2. С. 41-127.

6. Deng K., Levine H.A. The role of critical exponent in blow-up theorem: the sequel // J. Math. Anal. Appl. 2000. V. 243. P. 85-126.

7. John F. Blow-up of solutions of nonlinear wave equation in three space dimensions // Manuscripta Math. 1979. V. 28. P. 235-268.

8. Schaeffer J. The equation $u_{t t}-\Delta u=|u|^{p}$ for the critical value of $p / /$ Proc. Roy. Soc. Edinburgh A. 1985. V. 101. P. 31-44.

9. Sideris T. Nonexistence of global solutions to semilinear wave equations in high dimensions // J. Diff. Equations. 1984. V. 52. P. 378-406.

10. Del Santo D., Georgiev V., Mitidieri E. Global existence of the solutions and formation of singularities for a class of hyperbolic systems // Progress in Nonlinear Diff. Equations and Their Applications. V. 32. Boston: Birkhäuser, 1997. P. 117-140.

11. Дель Санто Д., Митидиери Э. Отсутствие решений гиперболической системы: критический случай // Дифференц. уравн. 1998. Т. 34. № 9. С. 1157-1163.

12. Kato T. Blow-up of solutions of some nonlinear hyperbolic equations // Comm. Pure. Appl. Math. 1980. V. 33. P. 501-505.

13. Veron L., Pohozaev S.I. Blow-up results for nonlinear hyperbolic inequalities // Ann. Scuola Norm. Sup. Pisa Cl. Sci. (4). 2000. V. 29. № 2. P. 393-420.

14. Курта В. В. Некоторые вопросы качественной теории нелинейных дифференциальных уравнений второго порядка: Дис. ... докт. физ.-мат. наук. М.: МИАН, 1994.

15. Митидиери Э., Похожаев С. И. Отсутствие положительных решений для квазилинейных эллиптических задач в $\mathbb{R}^{N}$ // Тр. МИАН. 1999. Т. 227. С. 192-222.

16. Митидиери Э., Похожсаев С.И. Априорные оценки и отсутствие решений дифференциальных неравенств в частных производных // Тр. МИАН. 2001. Т. 234.

17. Лаптев Г. Г. Об отсутствии решений одного класса сингулярных полулинейных дифференциальных неравенств // Тр. МИАН. 2001. Т. 232. С. 223-235.

18. Кондратьев $B$. A. Краевые задачи для эллиптических уравнений в областях с коническими и угловыми точками // Тр. Моск. матем. об-ва. 1967. Т. 16. С. 209-292.

19. Нгуен Мань Хунг. Асимптотика решений первой краевой задачи для сильно гиперболических систем вблизи конической точки границы области // Матем. сб. 2000. Т. 190. № 7. C. 103-126.

20. Бесов О.В., Ильин В. П., Никольский С. М. Интегральные представления функций и теоремы вложения. М.: Наука, 1996.

21. Zhang Q. Blow-up results for nonlinear parabolic equations on manifolds // Duke Math. J. 1999. V. 97. № 3. P. 515-539.

22. Лаптев Г. Г. Отсутствие глобальных положительных решений систем полулинейных эллиптических неравенств в конусах // Изв. РАН. Сер. матем. 2000. Т. 64. №6. C. 107-124.

23. Levine H. A. The role of critical exponents in blow-up theorems // SIAM Reviews. 1990. V. 32. P. 371-386. 